\title{
CDISC ADaM Parameter Type Terminology
}

National Cancer Institute

\section{Source}

National Cancer Institute. CDISC ADaM Parameter Type Terminology. NCI Thesaurus.

Code C81225.

Terminology associated with the Parameter Type codelist of the Clinical Data

Interchange Standards Consortium (CDISC) Analysis Data Model (ADaM). 